\title{
Genes de virulencia en Aeromonas spp. (Aeromonadales: Aeromonadaceae) aisladas de Oreochromis spp. (Perciformes: Cichlidae) para consumo humano en México
}

Jessica L. Ortega-Balleza, Alejandro Sánchez-Varela*, Isabel C. Rodríguez-Luna \& Xianwu Guo Laboratorio de Biotecnología Genómica, Centro de Biotecnología Genómica, Instituto Politécnico Nacional, Boulevard del Maestro S/N esq. Elías Piña, Col. Narciso Mendoza, CP. 88710, Cd. Reynosa, Tamaulipas, México; jessica_ortega7@hotmail.com, asanchezv@ipn.mx, isacrl@hotmail.com, gaopingguozhou@gmail.com * Correspondence

Recibido 23-III-2018. C Corregido 01-VIII-2018. Aceptado 20-IX-2018.

\begin{abstract}
Virulence genes in Aeromonas spp. (Aeromonadales: Aeromonadaceae) isolated from Oreochromis spp. (Perciformes: Cichlidae) destined for human consumption in Mexico. The genus Aeromonas are widely distributed in aquatic ecosystems are Gram-negative rods, oxidase-positive, and glucose-fermenting, considered emerging pathogens in humans. Aeromonas belongs to the fish microbiota, these microorganisms have a diversity of virulence factors responsible for a variety of infections in humans mainly gastrointestinal diseases. The presence of Aeromonas in products intended for consumption with high commercial demand such as tilapia generates sanitary concern due to the pathogenic potential of this bacteria. In this context, identification of virulence genes in strains of Aeromonas isolated in Oreochromis spp. intended for human consumption in Reynosa, Tamaulipas, Mexico is important due to the lack of molecular studies in this geographical area. In the present study the pathogenic potential of 15 strains of Aeromonas (A. veronii, A. hydrophila and A. schubertii) from Oreochromis spp. for human consumption were analyzed. Through PCR six virulence genes were analyzed (alt, ast, aerA, hlyA, gcat and stxl) and the strains used as control were: Aeromonas hydrophila subsp. hydrophila ATCC 7966, Aeromonas caviae 429865 INP, Escherichia coli O157: $\mathrm{H} 7$ and Escherichia coli $\mathrm{K} 12$. El $100 \%(\mathrm{n}=15)$ of the strains harbored at least one virulence gene, aerA gene was detected in $86.66 \%$ of the analyzed strains, while ast and $s t x 1$ genes were not identified. Moreover, Aeromonas strains had associated genes in the same strain: aerA / gcat, alt / aerA, alt / aerA / gcat / hlyA and alt / aerA / gcat, of which aerA / gcat were observed mostly in A. veronii, while A. hydrophila had the highest associations. These findings indicate that the strains of Aeromonas isolated in Oreochromis spp. have the potential to cause human diseases, and therefore, this species used as food, could be a vehicle for infections caused by Aeromonas. It also allows to provide information on this emerging microorganism to effectively treat and control any epidemiological event caused by Aeromonas spp. in the future.
\end{abstract}

Key words: Aeromonas; ecosystem; virulence genes; gastrointestinal; Oreochromis.

Ortega-Balleza, J. L., Sánchez-Varela, A., Rodríguez-Luna, I. C., \& Guo, X. (2018). Genes de virulencia en Aeromonas spp. (Aeromonadales: Aeromonadaceae) aisladas de Oreochromis spp. (Perciformes: Cichlidae) para consumo humano en México. Revista de Biología Tropical, 66(4), 1606-1613.

Las especies de Aeromonas son bacterias ubicuas de ecosistemas acuáticos, de morfología bacilar y Gram negativas, pueden ser mesófilas con movilidad o psicrófilas sin movilidad.
Esta bacteria es considerada un patógeno entérico emergente que además se asocia a infecciones de heridas, en sistema hepatobiliar, tracto respiratorio, tejidos blandos y septicemia 
en humanos (Janda \& Abbott, 2010; Figueras \& Beaz-Hidalgo, 2015; Batra Mathur, \& Misra, 2016).

El género Aeromonas posee una variedad de factores de virulencia como componentes estructurales y toxinas, además el género Aeromonas tiene la capacidad de capturar e integrar factores de virulencia en su genoma (Palma-Martínez et al., 2016) mismos que están involucrados en la colonización, invasión y proliferación de la bacteria conduciendo al desarrollo de la infección (Figueras \& Beaz-Hidalgo, 2015; Batra et al., 2016). Las manifestaciones clínicas en infecciones por Aeromonas sugieren que múltiples factores de virulencia actúan en conjunto, por lo que la virulencia podría ser específica de cada cepa que interviene evadiendo el sistema inmunitario mediante sus propias estrategias (Yu et al., 2005; Tomás, 2012).

A pesar de que algunos estudios han demostrado enterotoxicidad de algunas cepas de Aeromonas, no se ha establecido como un agente etiológico, ya que Aeromonas forma parte de las bacterias de difícil identificación bioquímica y comúnmente confundida por el género Vibrio (Castro-Escarpulli et al., 2003) por lo que los datos epidemiológicos de infecciones asociadas con esta bacteria son escasos y aunado también a la carencia de un modelo animal establecido para reproducir las infecciones causadas por Aeromonas (Tomás, 2012). Sin embargo, en 2004, ocurrió un brote de diarrea en Brasil, con 2170 casos, Aeromonas fue el patógeno aislado de 119 de 145 muestras. En 2012, en Brasil se reportó a Aeromonas en $2.7 \%$ a partir de muestras diarreicas aislando a $A$. caviae más frecuentemente seguida de $A$. hydrophila (Prediger et al., 2012). Mientras que, Tsheten et al., 2016, en el reino asiático de Bután reportaron un brote por intoxicación alimentaria causado por el consumo de carne con A. hydrophila.

Cabe señalar que Aeromonas es un microorganismo que forma parte de la microbiota de los peces, y estos productos alimenticios son de los más comercializados a nivel mundial y más de la mitad del valor de las exportaciones pesqueras procede de países en desarrollo (FAO, 2016). La tilapia es una especie cuya demanda a nivel mundial ha incrementado desde 1970, siendo el segundo grupo de peces más importante (Fuentes, Valladares, Grass, \& Pico, 2011). La presencia de Aeromonas en dichos productos puede causar enfermedades entéricas y por lo tanto son bacterias de interés para la salud pública, asimismo especies de Oreochromis spp. son endémicas en aguas del Río Bravo y canales que atraviesan la zona fronteriza de Reynosa, Tamaulipas. Por lo anterior, el objetivo de este estudio fue identificar genes de virulencia presentes en cepas de Aeromonas aisladas en Oreochromis spp. destinado a consumo humano en Reynosa, Tamaulipas, México.

\section{MATERIALES Y MÉTODOS}

Cepas de Aeromonas: En este estudio fueron analizadas 15 cepas de Aeromonas procedentes de un cepario del Laboratorio de Biotecnología Genómica del Centro de Biotecnología Genómica del Instituto Politécnico Nacional, dichas cepas fueron aisladas a partir de Oreochromis spp. destinadas a consumo humano y obtenidas entre junio y octubre de 2016 en aguas del Río Bravo y canales Rodhe y Anzaldúas que atraviesan la ciudad de Reynosa, Tamaulipas, México.

Identificación preliminar: a partir de las cepas almacenadas en glicerol al $85 \%$ a $-70{ }^{\circ} \mathrm{C}$, se tomaron $20 \mu \mathrm{l}$ y se inocularon en Agar Aeromonas, incubando a $37^{\circ} \mathrm{C}$ por $18 \mathrm{~h}$ para comprobar la pureza. Posteriormente, se procedió a realizar la identificación preliminar para lo cual las colonias típicas fueron sembradas en Agar Luria Bertani (IBI SCIENTIFIC) para después realizar las pruebas de tinción de Gram; actividad citocromo-c-oxidasa; actividad catalasa; oxidación-fermentación de la glucosa y hemolisis.

Identificación molecular: Se corroboró molecularmente la identidad de las cepas a través PCR del gen gyr $\mathrm{B}$ y secuenciación. 
Para lo cual las cepas bacterianas fueron inoculadas en caldo LB a $37{ }^{\circ} \mathrm{C}$ por $24 \mathrm{~h}$. El ADN genómico se extrajo con el kit Promega Wizard ${ }^{\circledR}$ Genomic Ref. A1120. Posteriormente se realizó una PCR del gen gyrB utilizando las condiciones reportadas por Sánchez-Varela, Ortega-Balleza, Rodríguez-Luna y Guo (2018) los productos obtenidos correspondían a 967 pb los cuales fueron secuenciados en el equipo ABI@3130 Genetic Analyzer, después las secuencias fueron analizadas en BLAST del NCBI para corroborar la especie a la cuál pertenecían. Se utilizaron como referencia las cepas Aeromonas hydrophila subsp. hydrophila ATCC 7966, E. coli O157:H7 y E. coli K12.

\section{Detección de genes de virulencia por Reacción en Cadena de la Polimerasa (PCR): La PCR de los genes de virulencia (alt, ast, aerA, hlyA, gcat y stx 1 ) se realizó a partir del ADNg extraído de las cepas de Aeromonas spp. Los iniciadores que se utilizaron se indi- can en el cuadro 1. La PCR se realizó a volu- men final de $25 \mu \mathrm{l}$; en cada tubo de reacción se colocó $1 \mu \mathrm{l} \mathrm{ADN}$ genómico (concentración promedio de 100-200 ng/ $\mu \mathrm{l}), 5 \mu \mathrm{l}$ de solución amortiguadora $1 \mathrm{X}, 0.75 \mu \mathrm{l}$ de $\mathrm{MgCl}_{2} 1.5 \mathrm{mM}$, $0.5 \mu \mathrm{l}$ dNTPs $0.05 \mathrm{mM}, 0.5 \mu \mathrm{l}$ de iniciadores 0.1 $\mu \mathrm{M}$ (Cuadro 1) y $1.25 \mathrm{U} / \mu \mathrm{l}$ de Taq polimerasa y $19 \mu \mathrm{l}$ de agua milli-Q estéril. La reacción se}

realizó en un termociclador Veriti ${ }^{\circledR}$ de Applied Biosystems, bajo las siguientes condiciones: 95 ${ }^{\circ} \mathrm{C}$ por $2 \mathrm{~min}$, enseguida 30 ciclos a $95{ }^{\circ} \mathrm{C}$ por $30 \mathrm{seg}$, la temperatura de alineamiento de cada uno de los iniciadores se muestra en el cuadro 1 , extensión a $72^{\circ} \mathrm{C}$ por $1.50 \mathrm{~min}$, y finalizar a $72{ }^{\circ} \mathrm{C}$ por $10 \mathrm{~min}$. Los productos resultantes fueron analizados mediante electroforesis en gel de agarosa $1.5 \%$ en solución amortiguadora de Tris Borato EDTA, pH 8 (TBE 1X), durante $1 \mathrm{~h}$ a 90 Volts. Después el gel fue visualizado en un fotodocumentador Kodak ${ }^{\circledR}$ con cámara Gel Logic 112.

Análisis estadístico: Los datos se analizaron usando el programa IBM ${ }^{\circledR}$ SPSS ${ }^{\circledR}$ Statistics versión 22 (SPSS Inc., Chicago, IL). La distribución de los genes de virulencia entre las especies de Aeromonas obtenidas se realizó mediante la prueba exacta de Fisher. Un valor de $\mathrm{P}<0.05$ se consideró estadísticamente significativo.

\section{RESULTADOS}

La identificación a nivel de especie mostró que, del total de cepas, 12 correspondían a $A$. veronii, dos a $A$. hydrophila y una correspondió a A. schubertii.

CUADRO 1

Iniciadores para detección de seis genes de virulencia en Aeromonas spp.

TABLE 1

Primers for the detection of six virulence genes of Aeromonas spp.

\begin{tabular}{|c|c|c|c|c|}
\hline Gen & Secuencia $\left(5^{\prime}-3^{\prime}\right)$ & Tamaño (pb) & $\operatorname{Tm}\left({ }^{\circ} \mathrm{C}\right)$ & Referencia \\
\hline $\begin{array}{l}\text { alt-F } \\
\text { alt-R }\end{array}$ & $\begin{array}{l}\text { tga ccc agt cet ggc acg gc } \\
\text { ggt gat cga tca cca cca gc }\end{array}$ & 442 & 64 & Nawaz et al., 2010 \\
\hline $\begin{array}{l}\text { ast-F } \\
\text { ast-R }\end{array}$ & $\begin{array}{l}\text { atc gtc agc gac agc ttc tt } \\
\text { ctc atc cet tgg ett gtt gt }\end{array}$ & 504 & 60 & Aguilera-Arreola et al., 2005 \\
\hline $\begin{array}{l}\text { aer-F } \\
\text { aer-R }\end{array}$ & $\begin{array}{l}\text { cet atg gec tga geg aga ag } \\
\text { cca gtt cca gtc cca cca ct }\end{array}$ & 431 & 64 & Soler et al., 2002 \\
\hline $\begin{array}{l}\text { hlyAF } \\
\text { hlyAR }\end{array}$ & $\begin{array}{l}\text { cca cgc aaa ttc atc acg } \\
\text { atc ctt gtt cac ctc gac }\end{array}$ & 1079 & 57 & Abdullah et al., 2003 \\
\hline $\begin{array}{l}\text { gcat-F } \\
\text { gcat-R }\end{array}$ & $\begin{array}{l}\text { ctc ctg gaa tcc caa gta tca } g \\
\text { ggc agg ttg aac agc agt atc t }\end{array}$ & 237 & 62 & Soler et al., 2002 \\
\hline $\begin{array}{l}\text { stx1F } \\
\text { stx1R }\end{array}$ & $\begin{array}{l}\text { agc agt cat tac ata aga ac } \\
\text { tgg att taa tgt cgc ata } g\end{array}$ & 144 & 53 & Hernández-Cortez et al., 2013 \\
\hline
\end{tabular}


CUADRO 2

Genes de virulencia detectados en cepas de Aeromonas

TABLE 2

Virulence genes detected in Aeromonas strains

$\begin{array}{ccccc}\text { Cepa / } & \text { A. veronii }(\mathrm{n}=12) & \text { A. hydrophila }(\mathrm{n}=2) & \text { A. schubertii }(\mathrm{n}=1) & \text { Total }(\mathrm{n}=15) \\ \text { Gen de virulencia } & 4 & 2 & 0 & 6 \\ \text { alt } & 0 & 0 & 0 & 0 \\ \text { ast } & 10 & 2 & 1 & 13 \\ \text { aerA } & 0 & 1 & 0 & 1 \\ \text { hlyA } & 6 & 2 & 0 & 8 \\ \text { gcat } & 0 & 0 & 0 & 0 \\ \text { stx } 1 & & \end{array}$

La identificación molecular de los genes de virulencia mostró que el $100 \%$ de las cepas de Aeromonas presentó al menos un gen de virulencia (Cuadro 2). El gen aerA se presentó en el $86.66 \%(13 / 15)$ y fue el más común. El gen gcat se detectó en $53.33 \%(8 / 13)$ de Aeromonas. Mientras que los genes alt y hlyA fueron identificados en $40.0(6 / 15)$ y $6.66 \%$ $(1 / 15)$ de las cepas. Por otra parte, tanto el gen ast como stx 1 no fueron identificados en este estudio. No hubo diferencias significativas entre la distribución de los genes alt, ast, aerA, hlyA, gcat y stx 1 , y las tres especies detectadas $(\mathrm{P}>0.05)$.

Asimismo, diferentes especies de Aeromonas llevan conjuntos distintos de genes de virulencia estudiados. En total se obtuvo que 53.33 $\%$ de Aeromonas presentaron más de un gen asociado en una misma cepa. En $A$. veronii se presentó prevalentemente aerA/gcat en $50.0 \%$ (3/6), alt/aerA $16.66 \%(1 / 6)$ y alt/aerA/gcat en $33.33 \%(2 / 6)$; mientras que en las dos cepas de A. hydrophila se observó tanto alt/aerA/gcat/ hlyA como alt/aerA/gcat. Sin embargo, en $A$. schubertii no fue detectada ninguna asociación.

\section{DISCUSIÓN}

Varios estudios han establecido un vínculo epidemiológico entre las fuentes de infección y los aislados clínicos de Aeromonas spp. (Hoel, Vadstein, \& Jakobsen, 2017), estudiar la composición bacteriana de productos alimenticios acuícolas es de vital importancia para evaluar la seguridad, riesgo de exposición a patógenos humanos y la adopción de medidas de control destinadas a disminuir la propagación de posibles bacterias patógenas (GrandeBurgos et al., 2018), ya que las enfermedades de transmisión alimentaria son un problema de salud mundial principalmente en países en vías de desarrollo (Tsheten et al., 2016).

Por otra parte, aunque el potencial de virulencia y los mecanismos patogénicos del género Aeromonas aún no están claros (Silva et al., 2017), se han estudiado factores de virulencia relacionados con la patogenicidad de la bacteria como estructuras y enzimas extracelulares, descritas principalmente en $A$. hydrophila (Castro-Escarpulli et al., 2002; Suárez \& Herrera, 2012) con la capacidad de dañar los tejidos del huésped y evadir su defensa inmune (Figueras \& Beaz-Hidalgo, 2015).

En este estudio, el gen aerA estuvo presente en mayor porcentaje ( $80 \%$ ) en las cepas analizadas, principalmente en $A$. veronii. A pesar de que esta enterotoxina es común en cepas clínicas de Aeromonas, estudios han mostrado que cepas ambientales también poseen dicho gen encontrándose en más del $80 \%$ de las cepas, las cepas analizadas en este trabajo proceden Oreochromis spp. de agua dulce, en dicha fuente este gen suele ser más prevalente (Ottaviani et al., 2011; Igbinosa \& Okoh, 2013; Abu-Elala, Abdelsalam, Marouf, \& Setta, 
2015). El gen aerA se presentó en $A$. veronii, A. hydrophila y A. shubertii que están asociadas a procesos infecciosos ya que la aerolisina (AerA), tiene actividad hemolítica y citotóxica, y causa daño extenso al epitelio (Chopra \& Houston, 1999).

Las lipasas son consideradas de importancia para la nutrición bacteriana, además de actuar como factores de virulencia (Onuk et al., 2013) y participar en la patogénesis en A. salmonicida (Chacón, Figueras, CastroEscarpulli, Soler, \& Guarro, 2003). En México, a partir de tilapia comercializada (Oreochromis niloticus niloticus) gcat estuvo presente en todos los aislados analizados (Castro-Escarpu1li et al., 2003). En Turquía, 2013, se obtuvo $36.6 \%$ de gcat a partir de aislados procedentes de pescado y agua superficial (Onuk et al., 2013), mientras que, los resultados obtenidos en este trabajo señalan que en $53.33 \%$ de las cepas evaluadas se detectó dicho gen, presentándose en $A$. veronii y $A$. hydrophila, cepas de alta frecuencia de detección en infecciones, no obstante, se esperaría encontrar a dicho gen en todas las cepas evaluadas ya que se considera especifico del género Aeromonas y ha llegado a ser de utilidad en la identificación del género (Khor, Puah, Tan, Puthucheary, \& Chua, 2015), en este estudio probablemente los cebadores empleados no son específicos para todas las cepas o estas presentan polimorfismos en esa región génica.

Por otra parte, en este estudio, el gen alt fue detectado tanto en $A$. veronii como $A$. hydrophila, otros trabajos también han reportado su presencia en diferentes especies (Ottaviani et al., 2011; Zhou et al., 2013), la enterotoxina citotónica termolábil (Alt) no provoca la degeneración de las criptas y las vellosidades intestinales, sin embargo, interviene en la aparición de diarrea secretora (Chopra \& Houston, 1999). Además, cepas positivas para el gen alt están asociadas con la aparición de heces blandas (Pablos, Rodríguez-Calleja, Santos, Otero, \& García-López, 2009). En este trabajo alt se detectó individualmente y también en asociación con otros genes incrementando la patogenicidad de Aeromonas, sin embargo, es importante destacar que, solo pueden expresarse en ciertas condiciones de crecimiento (Silva et al., 2017).

No se han encontrado reportes sobre la presencia de aerA/gcat en cepas de Aeromonas, cabe mencionar que tanto aer A como gcat son activados por serin proteasas (Aguilera-Arreola, Hernández-Rodríguez, Zúñiga, Figueras, \& Castro-Escarpulli, 2005) además de otras enzimas extracelulares, contribuyendo y potenciando la virulencia de Aeromonas (Nawaz et al., 2010). Aguilera-Arreola et al., 2007 reportaron $6.4 \%$ alt/aerA en cepas de A. veronii y A. caviae de España y México (Aguilera-Arreola et al., 2007). Además, no se ha establecido la relación entre la presencia de genes de toxinas específicos y la presentación clínica de la enfermedad (Hoel et al., 2017). Las combinaciones encontradas pueden depender de la región donde sea aislada la bacteria ya que además estan sometidas a diferentes presiones ambientales.

La incidencia de Aeromonas en las heces de pacientes con diarrea ha incrementado en los últimos años. En 2015, diferentes especies de Aeromonas fueron recuperadas de heces pacientes con gastroenteritis o diarrea aguda en la Ciudad de México, presentándose en cuarto lugar sólo por debajo de E. coli enteropatógena, Shigella y Salmonella, y por encima de bacterias que en diferentes países son frecuentemente causantes de enfermedades diarreicas como Campylobacter jejuni o Vibrio spp. (NovoaFarías et al., 2016), por lo cual la búsqueda de Aeromonas debe ser parte del diagnóstico de rutina en coprocultivos (Mendes-Marques et al., 2012).

En conclusión, estos hallazgos indican que las cepas de Aeromonas aisladas en Oreochromis spp. tienen el potencial de causar enfermedades en humanos, y por lo tanto este alimento podría ser vehículo de Aeromonas spp. Los resultados de este trabajo enfatizan la importancia de monitorear Aeromonas spp. en alimentos y con elevado potencial patogénico con la finalidad de contribuir en la aportación de información sobre este microrganismo emergente y para tratar y controlar eficazmente 
cualquier evento epidemiológico causado por Aeromonas spp. en el futuro.

Declaración de ética: los autores declaran que todos están de acuerdo con esta publicación y que han hecho aportes que justifican su autoría; que no hay conflicto de interés de cualquier tipo; y que han cumplido con todos los requisitos y procedimientos éticos y legales pertinentes. El documento firmado se encuentra en los archivos de la revista.

\section{AGRADECIMIENTOS}

Al Instituto Politécnico Nacional, a la Secretaria de Investigación y Posgrado, y a la Comisión de Operación y Fomento de Actividades Académicas del Instituto Politécnico Nacional (COFAA), asimismo al Programa Institucional de Formación de Investigadores (PIFI) por los recursos otorgados para la realización del presente trabajo de investigación.

\section{RESUMEN}

Las especies del género Aeromonas se encuentran ampliamente distribuidas en ecosistemas acuáticos, son bacilos Gram negativas, oxidasa positivas y fermentadoras de glucosa que han sido consideradas patógenas emergentes en humanos. Por otra parte, Aeromonas pertenece a la microbiota normal de los peces, no obstante, estos microorganismos poseen una diversidad de factores de virulencia responsables de una variedad de infecciones en humanos, principalmente de tipo gastrointestinal. La presencia de Aeromonas en productos destinados a consumo de alta demanda comercial como la tilapia genera preocupación sanitaria por el potencial patogénico que posee esta bacteria. En este contexto, identificar genes de virulencia presentes en cepas de Aeromonas aisladas en Oreochromis spp. para consumo humano en Reynosa, Tamaulipas, México; es de importancia ante la escasez de estudios moleculares al respecto en la zona. En el presente estudio se analizó el potencial patogénico de 15 cepas de Aeromonas previamente identificadas molecularmente mediante PCR y secuenciación, procedentes de Oreochromis spp. Mediante PCR se analizaron seis genes de virulencia (alt, ast, aerA, hlyA, gcat y stx 1$)$ y las cepas utilizadas como control fueron: Aeromonas hydrophila subsp. hydrophila ATCC 7966, Aeromonas caviae 429865 INP, Escherichia coli $\mathrm{O} 157: \mathrm{H} 7$ y Escherichia coli K12. El $100 \%(\mathrm{n}=15)$ de las cepas presentaron al menos un gen de virulencia, el gen aerA se detectó en $86.66 \%$ de las cepas analizadas, mientras que los genes ast y stx 1 no fueron identificados. Se encontró que las cepas de Aeromonas presentaban genes asociados en una misma cepa: aerA/gcat, alt/aerA, alt/ aer $\mathrm{A} /$ gcat/hlyA y alt/aer $\mathrm{A} / g c a t$, de los cuales aerA/gcat se observó con mayor frecuencia y principalmente en $A$. veronii, mientras que, $A$. hydrophila presentó el mayor número de asociaciones de genes de virulencia. Estos hallazgos indican que las cepas de Aeromonas aisladas en Oreochromis spp. tienen el potencial de causar enfermedades en humanos. Por lo tanto, es necesario proporcionar información sobre esta bacteria emergente, para tratar y controlar eficazmente cualquier posible evento epidemiológico causado por la misma.

Palabras clave: Aeromonas; ecosistema; humanos; genes de virulencia; gastrointestinal; Oreochromis.

\section{REFERENCIAS}

Abdullah, A. I., Hart, C. A., \& Winstanley, C. (2003). Molecular characterization and distribution of virulence-associated genes amongst Aeromonas isolates from Libya. Journal of Applied Microbiology, 95(5), 1001-1007.

Abu-Elala, N., Abdelsalam, M., Marouf, S., \& Setta, A. (2015). Comparative analysis of virulence genes, antibiotic resistance and gyrB-based phylogeny of motile Aeromonas species isolates from Nile tilapia and domestic fowl. Letters in Applied Microbiology, 61(5), 429-436.

Aguilera-Arreola, M. G., Hernández-Rodríguez, C., Zúñiga, G., Figueras, M. J., \& Castro-Escarpulli, G. (2005). Aeromonas hydrophila clinical and environmental ecotypes as revealed by genetic diversity and virulence genes. FEMS Microbiology Letters, 242(2), 231-240.

Aguilera-Arreola, M. G., Hernández-Rodríguez, C., Zúñiga, G., Figueras, M. J., Garduño, R. A., \& CastroEscarpulli, G. (2007). Virulence potential and genetic diversity of Aeromonas caviae, Aeromonas veronii, and Aeromonas hydrophila clinical isolates from Mexico and Spain: a comparative study. Canadian Journal of Microbiology, 53(7), 877-87.

Batra, P., Mathur, P., \& Misra, M. C. (2016). Aeromonas spp.: An Emerging Nosocomial Pathogen. Journal of Laboratory Physicians, 8(1), 1-4.

Castro-Escarpulli, G., Aguilera-Arreola, M. G., GionoCerezo, S., Hernández-Rodríguez, C. H., RodríguezChacón, M., Soler-Falgas, L., ... Figueras-Salvat, M. J. (2002). El género Aeromonas. ¿Un patógeno importante en México? Enfermedades Infecciosas y Microbiología, 22(4), 1-12.

Castro-Escarpulli, G., Figueras, M. J., Aguilera-Arreola, G., Soler, L., Fernández-Rendón, E., Aparicio, G. O., ... Chacón, M. R. (2003). Characterisation of 
Aeromonas spp. isolated from frozen fish intended for human consumption in Mexico. International Journal of Food Microbiology, 84(1), 41-49.

Chacón, M. R., Figueras, M. J., Castro-Escarpulli, G., Soler, L., \& Guarro, J. (2003). Distribution of virulence genes in clinical and environmental isolates of Aeromonas spp. Antonie van Leeuwenhoek, 84, 269-278.

Chopra, A. K., \& Houston, C. W. (1999). Enterotoxins in Aeromonas-associated gastroenteritis. Microbes and Infection, 1(13), 1129-1137.

FAO. (2016). El estado mundial de la pesca y la acuicultura. Roma, Italia: FAO.

Figueras, M. J., \& Beaz-Hidalgo, R. (2015). Aeromonas infections in humans. In J. Graf (Ed.), Aeromonas (pp. 65-108). Norfolk, GB: Caister Academic Press.

Fuentes, M., Valladares, J., Grass, G., \& Pico, Y. (2011). Microbiota de interés para la salud pública de Oreochromis spp. (tilapia roja) cultivada en jaulas flotantes en agua dulce. Revista Cubana de Investigaciones Pesqueras, 28(2), 74-80.

Grande-Burgos, M. J., Romero, J. L., Pérez-Púlido, R., Cobo-Molinos, A., Galvez, A., \& Lucas, R. (2018). Analysis of potential risks from the bacterial communities associated with air-contact surfaces from tilapia (Oreochromis niloticus) fish farming. Environmental Research, 160, 385-390.

Hernández-Cortez, C., Méndez-Tenorio, A., AguileraArreola, M. G., \& Castro-Escarpulli, G. (2013). Design and standardization of four multiplex polymerase chain reactions to detect bacteria that cause gastrointestinal diseases. African Journal of Microbiology Research, 7(21), 2673-2681.

Hoel, S., Vadstein, O., \& Jakobsen, A. N. (2017). Species Distribution and Prevalence of Putative Virulence Factors in Mesophilic Aeromonas spp. Isolated from Fresh Retail Sushi. Frontiers in Microbiology, 8, 931.

Igbinosa, I. H., \& Okoh, A. I. (2013). Detection and distribution of putative virulence associated genes in Aeromonas species from freshwater and wastewater treatment plant. Journal of Basic Microbiology, 53(11), 895-901.

Janda, J. M., \& Abbott, S. L. (2010). The genus Aeromonas: Taxonomy, pathogenicity, and infection. Clinical Microbiology Reviews, 23(1), 35-73.

Khor, W. C., Puah, S. M., Tan, J., Puthucheary, S., \& Chua, K. H. (2015). Phenotypic and Genetic Diversity of Aeromonas Species Isolated from Fresh Water Lakes in Malaysia. PLoS ONE, 10(12), e0145933.

Mendes-Marques, C. L., Nascimento, L. M., Theophilo, G. N. D., Hofer, E., Melo-Neto, O. P., \& Leal, N.
C. (2012). Molecular characterization of Aeromonas spp. and Vibrio cholerae O1 isolated during a diarrhea outbreak. Revista do Instituto de Medicina Tropical de São Paulo, 54(6), 299-304.

Nawaz, M., Khan, S. A., Khan, A. A., Sung, K., Tran, Q., Kerdahi, K., \& Steele, R. (2010). Detection and characterization of virulence genes and integrons in Aeromonas veronii isolated from catfish. Food Microbiology, 27(3), 327-331.

Novoa-Farías, O., Frati-Munari, A. C., Peredo, M. A., Flores-Juárez, S., Novoa-García, O., Galicia-Tapia, J., \& Romero-Carpio, C. E. (2016). Susceptibilidad de las bacterias aisladas de infecciones gastrointestinales agudas a la rifaximina y otros agentes antimicrobianos en México. Revista de Gastroenterologia de Mexico, 81(1), 3-10.

Onuk, E. E., Findik, A., Turk, N., Altun, S., Korun, J., Ozer, S., ... Ciftci, A. (2013). Molecular identification and determination of some virulence genes of Aeromonas spp. in fish and water from Turkish coastal regions. Revista de Medicina Veterinaria, 164, 200-206.

Ottaviani, D., Parlani, C., Citterio, B., Masini, L., Leoni, F., Canonico, C., ... Pianetti, A. (2011). Putative virulence properties of Aeromonas strains isolated from food, environmental and clinical sources in Italy: A comparative study. International Journal of Food Microbiology, 144(1), 538-545.

Pablos, M., Rodríguez-Calleja, J. M., Santos, J. A., Otero, A., \& García-López, M. L. (2009). Occurrence of motile Aeromonas in municipal drinking water and distribution of genes encoding virulence factors. International Journal of Food Microbiology, 135(2), 158-164.

Palma-Martínez, I., Guerrero-Mandujano, A., Ruiz-Ruiz, M. J., Hernández-Cortez, C., Molina-López, J., Bocanegra-García, V., \& Castro-Escarpulli, G. (2016). Active shiga-like toxin produced by some Aeromonas spp., isolated in Mexico City. Frontiers in Microbio$\log y, 7(1), 1-7$.

Prediger, K. de C., Pereira, R. da S., Neto, C. H. del P. W., Santos, R. C. V., Fadel-Picheth, C. M. T., \& Vizzotto, B. S. (2012). A prospective study on Aeromonas in outpatients with diarrhea in the central region of Rio Grande do Sul State. Brazilian Journal of Microbio$\log , 43(3), 966-968$.

Sánchez-Varela, A., Ortega-Balleza, J. L., Rodríguez-Luna, I. C., \& Guo, X. (2018). Molecular identification of Aeromonas spp. isolated from water in northeastern México. ECORFAN Journal-Bolivia, 5-8, 14-8.

Silva, L. C. A. da, Leal-Balbino, T. C., Melo, B. S. T. de, Mendes-Marques, C. L., Rezende, A. M., Almeida, A. M. P. de, \& Leal, N. C. (2017). Genetic diversity and virulence potential of clinical and environmental Aeromonas spp. isolates from a diarrhea outbreak. BMC Microbiology, 17, 179. 
Soler, L., Figueras, M. J., Chacón, M. R., Vila, J., Marco, F., Martínez-Murcia, A. J., \& Guarro, J. (2002). Potential virulence and antimicrobial susceptibility of Aeromonas popoffii recovered from freshwater and seawater. FEMS Immunology and Medical Microbiology, 32(3), 243-247.

Suárez, Q. W. \& Herrera, A. F. (2012). Determinación de factores de virulencia en cepas de Aeromonas spp. Revista MVZ Córdoba, 17(1), 2846-2851.

Tomás, J. M. (2012). The main Aeromonas pathogenic factors. ISRN Microbiology, 2012, 1-22.

Tsheten, T., Tshering, D., Gyem, K., Dorji, S., Wangchuk, S., Tenzin, T., ... Jamtsho, T. (2016). An Outbreak of
Aeromonas hydrophila Food Poisoning in Deptsang Village, Samdrup Jongkhar, Bhutan. Journal of Research in Health Sciences, 16(4), 224-227.

Yu, H. B., Zhang, Y. L., Lau, Y. L., Yao, F., Vilches, S., Merino, S., ... Leung, K. Y. (2005). Identification and characterization of putative virulence genes and gene clusters in Aeromonas hydrophila PPD134 / 91. Applied Environmental Microbiology, 71(8), 4469-4477.

Zhou, Q. L., Wang, Y. J., Xie, J., Ge, X. P., Xi, B. W., \& Liu, B. (2013). Distribution and virulence gene comparison of Aeromonas strains isolated from diseased fish and water environment. Polish Journal of Microbiology, 62(3), 299-302. 